\title{
Use of Handheld Ultrasound Device with Artificial Intelligence for Evaluation of Cardiorespiratory System in COVID-19
}

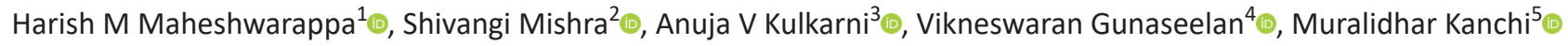

\begin{abstract}
Background: Coronavirus disease-2019 (COVID-19) causes various cardiopulmonary manifestations. Bedside ultrasound helps in the rapid diagnosis of these manifestations. Vscan Extend ${ }^{T M}$ (GE, Wauwatosa, WI, USA) is a handheld ultrasound device with a dual probe and an artificial intelligence application to detect ejection fraction. It can help in reducing the time for diagnosis, duration, and the number of healthcare workers exposed to COVID-19. This is a prospective observational study comparing the cardiorespiratory parameters and time duration for assessment between Vscan Extend ${ }^{\mathrm{TM}}$ and the conventional ultrasound machine.

Materials and methods: Paired observations were made in 96 COVID-19 patients admitted to the intensive care unit by two intensivists. Intensivist A used the Vscan Extend ${ }^{\text {TM }}$ device to assess the cardiac function, lung fields, diaphragm, deep veins, and abdomen. Intensivist B used clinical examination, X-ray chest, ECG, and conventional echocardiogram for assessment. The agreement between the findings and the time duration required in both the methods was compared.

Results: The use of handheld ultrasound has significantly decreased the duration of bedside examination of patients than the conventional method. The median duration of examination using handheld ultrasound was 9 (8.0-11.0) minutes, compared to 20 (17-22) minutes with the conventional method $(P<0.001)$. The Cohen's kappa coefficient was 1.0 for left ventricular systolic function, most of the lung fields, and diaphragmatic movement.

Conclusion: Vscan Extend ${ }^{\mathrm{TM}}$ helps in the rapid identification and diagnosis of cardiopulmonary manifestations in COVID-19 patients. The agreement between the handheld device and the conventional method proves its efficacy and safety.

CTRI Number: CTRI/2020/07/026701

Keywords: Artificial intelligence, COVID-19, Critically ill; Handheld ultrasound devices, Point of care, Ultrasound.

Indian Journal of Critical Care Medicine (2021): 10.5005/jp-journals-10071-23803
\end{abstract}

\section{INTRODUCTION}

Coronavirus disease-2019 (COVID-19), caused by SARS CoV2, has varied clinical manifestations. Over $85 \%$ of the infected population remain asymptomatic, while approximately $4 \%$ present with severe respiratory distress syndrome requiring admission to the intensive care unit. ${ }^{1}$ Extrapulmonary presentations like acute coronary syndrome, congestive cardiac failure, and thromboembolism are also well known in COVID-19. ${ }^{2}$ In the background of the COVID-19 pandemic, any patient attending the emergency department, the COVID-19-induced pneumopathy, cardiac abnormality, and thromboembolism should be ruled out. ${ }^{3}$ The virus spread occurs by aerosol route. ${ }^{4}$ Involving more than one person for diagnosis and management at this pandemic increases the risk of infection spread among healthcare workers. A bedside ultrasound is a useful tool to help a primary physician or an intensivist to screen the patient. If the diagnosis and management need expert advice and consultation, they can be called over. This approach reduces the chance of infection spread among healthcare workers and reduces the burden on the exhausted healthcare system due to pandemic. ${ }^{5}$ Handheld ultrasound is of more significant application compared to conventional ultrasound in critical care. The image quality and resolution are equally good comparing to conventional ultrasound and echocardiography machines. ${ }^{6-8}$ It is easy to carry around like a stethoscope, has low cost, and is easier to disinfect than conventional ultrasound machine.

\footnotetext{
1,2Department of Critical Care Medicine, Mazumdar Shaw Medical Centre, Narayana Health City, Bengaluru, Karnataka, India

${ }^{3}$ Narayana Institute of Cardiac Sciences, Narayana Health City, Bengaluru, Karnataka, India

${ }^{4}$ Department of Clinical Research, Narayana Hrudayalaya Limited, Bengaluru, Karnataka, India

${ }^{5}$ Department of Anaesthesiology and Intensive Care, Narayana Institute of Cardiac Sciences, Bengaluru, Karnataka, India

Corresponding Author: Harish M Maheshwarappa, Department of Critical Care Medicine, Mazumdar Shaw Medical Centre, Narayana Health City, Bengaluru, Karnataka, India, Phone: +91 8095218493, e-mail: dr.harishmm@rocketmail.com
}

How to cite this article: Maheshwarappa HM, Mishra S, Kulkarni AV, Gunaseelan V, Kanchi M. Use of Handheld Ultrasound Device with Artificial Intelligence for Evaluation of Cardiorespiratory System in COVID-19. J Crit Care Med 2021;25(5):524-527.

Source of support: Nil

Conflict of interest: None

Vscan Extend ${ }^{\mathrm{TM}}$ from General Electric Healthcare (GE, Wauwatosa, WI, USA), a handheld ultrasound device launched with a dual ultrasound probe. The dual probe has a phased array probe (1.5-3.8 MHz) for bedside transthoracic echocardiography. The other end has a linear probe $(3.5-8 \mathrm{MHz})$, which can be used 
for lung ultrasound, duplex sonography of the venous system, and central venous cannulation. This device also has an artificial intelligence application. After acquiring an apical four-chamber without foreshortening the apex, the application automatically traces the endocardial border of the left ventricle. Thus it detects the left ventricular end-diastolic volume (LVEDV) and left ventricular end-systolic volume (LVESV). The ejection fraction (EF) of the left ventricle is calculated from these two values. Thus, the study was conducted with the primary aim to assess the effect of Vscan Extend ${ }^{\mathrm{TM}}$ handheld ultrasound device on the duration of cardiopulmonary assessment in COVID-19 patients. We also assessed the efficacy and safety profile of this new generation handheld ultrasound machine by comparing its findings with the conventional ultrasound machine.

\section{Materials and Methods}

In a prospective observational study conducted in COVID-19 intensive care unit at a tertiary care medical institution, over 1 month in August 2020, after obtaining approval from the institutional ethics committee, all critically ill COVID-19-positive patients above 18 years of age were included. Pediatric patients (age $<18$ years) and patients with psychiatric problems were excluded from the study. After obtaining informed written consent, patients were evaluated using handheld ultrasound device Vscan Extend $^{\mathrm{TM}}$, General Electric Healthcare (GE, Wauwatosa, WI, USA), by an intensivist, adequately trained in bedside ultrasound (intensivist A). Intensivist A was blinded to the clinical diagnosis of the patient. Lung ultrasound was done using the eight-segment technique, evaluating each segment for pleural sliding, pleural irregularities, B-lines, and consolidation. ${ }^{9}$ Findings were be entered for each segment in the proforma based on lung ultrasound. Diaphragmatic movement and presence of pleural fluid and abdominal free fluid collection were noted. Cardiac ultrasound was done using a phased array probe. Apical four-chamber, apical two-chamber, parasternal short axis, and long-axis views were assessed. Any regional wall motion abnormality, pericardial effusion, and right heart dysfunction were documented. Left ventricular EF was calculated in the apical four-chamber view using LVivo application in Vscan Extend ${ }^{\mathrm{TM}}$ machine (Fig. 1). Color Doppler was applied across

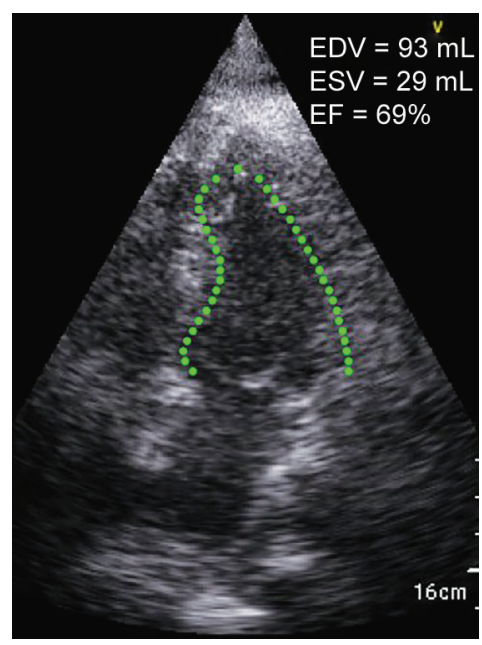

Fig. 1: Image showing use of LVivo application of Vscan Extend ${ }^{\mathrm{TM}}$ to calculate EF aortic, mitral, tricuspid, and pulmonary valves to look for any flow turbulence. Any valvular pathology was documented. inferior vena cava (IVC) collapsibility index was calculated from the subcostal view. The total duration of examination and duration of cardiac ultrasound was documented. The other intensivist trained in point of care ultrasound (intensivist B) performed clinical examination and point of care cardiac ultrasound with a standard ECHO machine (Vivid, GE). The patient's respiratory system was examined using bedside ultrasound with multiple probes, chest X-ray, and auscultation for added sounds (crepitations and bronchial breathing), reduced air entry, and diaphragm movement. The cardiac examination was done by auscultation, especially for added sounds, murmurs, elevated Jugular venous pressure, and reduced cardiac sounds, and ECG. Any regional wall motion abnormality, right heart dysfunction, pericardial effusion, valvular pathology, IVC collapsibility were identified using Vivid, GE echo machine. Left ventricular EF was calculated in apical four-chamber view using Simpson's method. The abdomen and lower limb were examined clinically. The total time of clinical examination was noted.

At the end of the study, the agreement between the intensivists' diagnostic findings was compared statistically. Assessment of the level of agreement between both groups was done using the Cohen's kappa coefficient. Lung zones were assessed qualitatively on the basis of the presence of pneumothorax, consolidation, or edema, and the agreement between handheld and conventional ultrasound device findings was assessed using Cohen's kappa coefficient.

Sample size was calculated using nMaster software v2.0. Keeping a null hypothesis agreement as 0.5 and an alternative hypothesis agreement as 0.8 with prevalence of findings as $25 \%$, power $80 \%$, and $5 \%$ alpha error the minimum required sample size was 84 patients. Here, prevalence refers to the proportion of patients expected to have abnormal lung or cardiac findings based on your objective. ${ }^{2}$

\section{Results}

In this prospective study, a total of 96 paired readings using two different methods were obtained by two intensivists, blinded to each other's findings. The demographic characteristics of the patients are discussed in Table 1. The use of handheld ultrasound has significantly decreased the time required for bedside examination of patients than the conventional method. The median duration of examination using handheld ultrasound was 9 (8.0-11.0) minutes, compared to 20 (17-22) minutes with the conventional method $(P<0.001)$ (Table 2$)$. The agreement was perfect on comparing left ventricular (LV) systolic function with a Cohen's kappa coefficient of 1.0 (Table 3). Both the groups show moderate agreement for regional wall motion abnormality (RWMA) with a coefficient of 0.53 $[0.37,0.69]$. The mean EF obtained with the handheld ultrasound was $45.89 \pm 11.4(\%)$, with LVESV and LVEDV of $46.28 \pm 11.8 \mathrm{~mL}$ and $46.28 \pm 11.8 \mathrm{~mL}$, respectively. We found a poor agreement for right ventricular (RV) systolic function and pericardial effusion between the two groups with a coefficient of 0.07 and -0.01 , respectively. Cohen's kappa coefficient showed an agreement ranging from substantial to perfect for lung parameters between the two groups (Table 4). Among other parameters, we observed fair agreement in IVC collapsibility between both the groups, with a coefficient of $0.37[0.25,0.49]$. We found substantial agreement between both groups for abdominal free fluid collection with a coefficient of $0.78[0.55,1.0]$. 
Use of Artificial Intelligence in COVID-19 Management

Table 1: Demographic characteristics

\begin{tabular}{|c|c|}
\hline Age (years) & $55.13 \pm 14.6$ \\
\hline \multicolumn{2}{|l|}{ Sex } \\
\hline Male $(n, \%)$ & 70 (72.9\%) \\
\hline Female $(n, \%)$ & $26(27.1 \%)$ \\
\hline \multicolumn{2}{|l|}{ Diagnostic test } \\
\hline RT PCR (n, \%) & 59 (61.5\%) \\
\hline $\mathrm{CT}(\mathrm{n}, \%)$ & $0(0.0 \%)$ \\
\hline TruNat (n, \%) & 19 (19.8\%) \\
\hline Rapid antigen (n, \%) & $17(17.7 \%)$ \\
\hline Height (cm) & $171.02 \pm 6.6$ \\
\hline Weight (kg) & $80.0[90.0-71.25]$ \\
\hline HR (rate/min) & $92.03 \pm 23.9$ \\
\hline $\mathrm{SpO}_{2}(\%)$ & $94.54 \pm 4.0$ \\
\hline RR (breaths/min) & $26.58 \pm 5.4$ \\
\hline $\mathrm{SBP}(\mathrm{mmHg})$ & $129.93 \pm 21.3$ \\
\hline $\mathrm{DBP}(\mathrm{mmHg})$ & $70.09 \pm 14.1$ \\
\hline MAP $(\mathrm{mmHg})$ & $90.63 \pm 15.9$ \\
\hline \multicolumn{2}{|l|}{ Intubation } \\
\hline Absent (n, \%) & $46(47.9 \%)$ \\
\hline Present (n, \%) & $50(52.1 \%)$ \\
\hline \multicolumn{2}{|l|}{ Mode } \\
\hline PCV $(n, \%)$ & $2(2.1 \%)$ \\
\hline $\operatorname{PRVC}(n, \%)$ & $16(16.7 \%)$ \\
\hline PCV-VG $(n, \%)$ & $24(25.0 \%)$ \\
\hline $\operatorname{SIMV}(n, \%)$ & $0(0.0 \%)$ \\
\hline PSV (n, \%) & $8(8.3 \%)$ \\
\hline PEEP $(\mathrm{cmH} 2 \mathrm{O})$ & $8.12 \pm 2.5$ \\
\hline $\mathrm{FiO}_{2}(\%)$ & $60.02 \pm 21.8$ \\
\hline$P_{\text {peak }}(\mathrm{cmH} 2 \mathrm{O})$ & $30.04 \pm 7.9$ \\
\hline$P_{\text {mean }}(\mathrm{cmH} 2 \mathrm{O})$ & $15.66 \pm 4.8$ \\
\hline Proned & 47 (48.9\%) \\
\hline
\end{tabular}

$\mathrm{HR}$, heart rate; $\mathrm{SpO}_{2}$, oxygen saturation; $\mathrm{RR}$, respiratory rate; $\mathrm{SBP}$, systolic blood pressure; DBP, diastolic blood pressure; MAP, mean arterial pressure; $P C V$, pressure control ventilation; PRVC, pressure regulated volume control; PCV-VG, pressure control ventilation-volume guaranteed; SIMV, synchronized intermittent mandatory ventilation; PSV, pressure support ventilation, $\mathrm{PEEP}$, peak end expiratory pressure; $\mathrm{FiO}_{2}$, fraction of inspired oxygen; $P_{\text {peak, }}$ peak airway pressure; $P_{\text {mean }}$, mean airway pressure; RT PCR, reverse transcription polymerase chain reaction; $\mathrm{CT}$, computed tomography

Table 2: Comparison of time duration between two groups

\begin{tabular}{llll}
\hline & $\begin{array}{l}\text { Conventional } \\
\text { group }(N=96)\end{array}$ & $\begin{array}{l}\text { Handheld ultrasound } \\
\text { group }(N=96)\end{array}$ & Pvalue \\
\hline $\begin{array}{l}\text { Total duration } \\
\text { (minutes) }\end{array}$ & $20.0[22.0-17.0]$ & $9.0[11.0-8.0]$ & $<0.001$ \\
Median & & & \\
[Q3-Q1] & & & \\
\hline$P<0.05:$ staistion
\end{tabular}

$P<0.05$ : statistically significant; statistical method used: Mann-Whitney $\mathrm{U}$ test

\section{Discussion}

In this prospective observational study, we found significant agreement between the cardiac and lung measured parameters obtained with handheld equipment and conventional ultrasound machine in COVID-19 patients. Along with the agreement in both
Table 3: Comparison of cardiac findings between both the groups

\begin{tabular}{lll}
\hline Cardiac parameter & $\begin{array}{l}\text { Cohen's kappa value } \\
\text { with 95\% of Cl }\end{array}$ & P value \\
\hline LVsys & $1.0[1.0,1.0]$ & $<0.001$ \\
RVsys & $0.07[-0.16,0.29]$ & $<0.56$ \\
Pericardial effusion & $-0.01[-0.03,0.004]$ & 0.16 \\
RWMA & $0.53[0.37,0.69]$ & $<0.001$ \\
Valve regurgitation & $0.02[-0.10,0.14]$ & 0.79 \\
\hline
\end{tabular}

LVsys, left ventricular systolic function; RVsys, right ventricular systolic function; RWMA, regional wall motion abnormality; $P<0.05$ : statistically significant; statistical method used: Cohen's kappa agreement

Table 4: Comparison of lung findings between both the groups

\begin{tabular}{lll}
\hline Lung parameter & $\begin{array}{l}\text { Cohen's kappa value } \\
\text { with 95\% of Cl }\end{array}$ & Pvalue \\
\hline Right lateral upper zone & $1.0[1.0,1.0]$ & $<0.001$ \\
Right anterior upper zone & $0.94[0.88,1.0]$ & $<0.001$ \\
Left anterior upper zone & $1.0[1.0-1.0]$ & $<0.001$ \\
Left lateral upper zone & $0.76[0.64,0.87]$ & $<0.001$ \\
Right lateral lower zone & $0.67[0.54,0.80]$ & $<0.001$ \\
Right anterior lower zone & $0.98[0.95,1.0]$ & $<0.001$ \\
Left anterior lower zone & $1.0[1.0,1.0]$ & $<0.001$ \\
Left lateral lower zone & $1.0[1.0,1.0]$ & $<0.001$ \\
Pleural effusion & $1.0[1.0,1.0]$ & $<0.001$ \\
Diaphragmatic dysfunction & $1.0[1.0,1.0]$ & $<0.001$ \\
\hline
\end{tabular}

$P<0.05$ : statistically significant; statistical method used: Cohen's kappa agreement

the groups, a statistically significant difference was observed in the time required for bedside examination using handheld ultrasound compared to the conventional method. This reduction in time for bedside examination and accurate measurement reduces the duration of exposure and the number of healthcare workers getting exposed to the COVID-19 patients.

Various studies have evaluated the role of handheld ultrasound in different aspects of critical care in the past. Its role has been identified in both diagnostic and therapeutic settings. Handheld ultrasound is of more significant application in cardiac critical care. COVID-19 infection is associated with hyperdynamic cardiac function, stress-induced cardiomyopathy, RV enlargement, acute pulmonary hypertension, and diffuse myocardial inhibition. ${ }^{3}$ Studies have shown good agreement between handheld ultrasound and conventional echocardiography for diagnosing pericardial effusion, RWMA, valvular pathologies, and left ventricular EF. In a study comparing hand-carried ultrasound and conventional echocardiography, Tsutsui et al. showed moderate agreement between both groups for LV function and RWMA ( $k=0.58)$. They also showed a good correlation $(\kappa=0.85)$ for valvular regurgitation, while stenotic pathologies could not be determined because of the technical limitation of hand-carried ultrasound. ${ }^{6}$ Our study showed a similar agreement as to the previous study for RWMA $(\kappa=0.53)$ and a perfect agreement for LV systolic function $(\kappa=1.0)$. The poor agreement seen in our study for valvular regurgitation $(\kappa=0.02)$ and RV systolic function $(\kappa=0.07)$ might be due to poor echocardiography window caused by chest wall edema in prone patients. 
Similarly, lung ultrasound has excellent sensitivity for detecting various lung pathologies such as pneumothorax, interstitial edema, consolidation, pleural effusion, and diaphragmatic dysfunction. ${ }^{10}$ Lung ultrasound is as useful in diagnosing lung pathologies as a routine clinical examination and bedside chest $\mathrm{X}$ ray. ${ }^{11}$ Common lung ultrasound findings seen in COVID-19 patients are B-lines, air bronchograms, consolidation, and pleural irregularities. ${ }^{10} \mathrm{We}$ found a strong agreement for the above findings in six out of eight lung zones between both the groups ( $\kappa=0.94-1.0)$. Also, a strong agreement was seen for pleural effusion and diaphragmatic movement $(K=1.0)$. Kajimoto et al., in a study comparing handheld ultrasound to conventional methods, showed the highest specificity, negative, positive predictive value, and accuracy with handheld ultrasound for rapidly diagnosing acute heart failure syndrome and evaluating acute dyspnea. Though they did not compare the total duration required for different methods. ${ }^{13}$ Our study found a statistically significant reduction in the time required for bedside examination ( 9.0 vs. 20.0 minutes) using handheld ultrasound with high accuracy of the findings. Handheld ultrasound has higher sensitivity in assessing abdominal free fluid in hepatorenal recess than the conventional ultrasound machine. ${ }^{8}$ Our study also found a substantial to perfect agreement between handheld ultrasound and conventional methods for abdominal free fluid $(K=1.0)$.

Factors like the presence of arrhythmias, extremes of heart rate, COPD patients, chest wall edema in the prone patient may lead to the poor echocardiographic window and interfere with measurement and evaluation by handheld ultrasound. We should keep this limitation in mind.

The SARS-CoV2 virus is highly contagious and has aerosol spread as well. ${ }^{14}$ The use of routine imaging studies is discouraged by the American College of Radiology. ${ }^{15}$ Various types of literature have discussed the role of handheld ultrasound in the COVID-19 pandemic. ${ }^{14,16,17}$ However, there is a scarcity of studies evaluating the role, accuracy, and rapidity of measurement by handheld ultrasound in the COVID-19 pandemic.

This study has shown the high accuracy of measurements by handheld ultrasound, with a high agreement with the conventional method and the rapidity of its assessment.

\section{Conclusion}

Vscan Extend ${ }^{\mathrm{TM}}$ handheld ultrasound device helps in the rapid identification, assessment, and diagnosis of cardiopulmonary manifestations in COVID-19 patients. The agreement between the Vscan Extend ${ }^{\mathrm{TM}}$ handheld ultrasound device and the conventional method proves its efficacy and safety. Use of Vscan Extend ${ }^{\text {TM }}$ handheld ultrasound device in daily use on a larger perspective both in COVID-19 patients and otherwise may reduce the burden on the healthcare system by assisting in rapid diagnosis and involvement of less healthcare personnel for cardiopulmonary assessment.

\section{Highlights}

This research article highlights the role of Vscan Extend ${ }^{\mathrm{TM}}$ handheld ultrasound device with artificial intelligence in rapid assessment of cardiopulmonary status in COVID-19 patients, hence reducing the burden on healthcare workers, while being accurate and safe.

\section{ORCID}

Harish M Maheshwarappa @ https://orcid.org/0000-0001-9377-3421 Shivangi Mishra ๑ https://orcid.org/0000-0003-4960-2400 Anuja V Kulkarni @ https://orcid.org/0000-0002-8540-0024
Vikneswaran Gunaseelan @ https://orcid.org/0000-0002-8191-1572 Muralidhar Kanchi i https://orcid.org/0000-0003-3347-0204

\section{References}

1. Halbach JL, Prieto JM, Wang AW, Hawisher D, Cauvi DM, Reyes T, et al. Early hyperbaric oxygen therapy improves survival in a model of severe sepsis. Am J Physiol Integr Comp Physiol 2019;317(1):R160R168. DOI: 10.1152/AJPREGU.00083.2019.

2. Adukia SA, Ruhatiya RS, Maheshwarappa HM, Manjunath RB, Jain GN. Extrapulmonary features of COVID-19: a concise review. Indian J Crit Care Med 2020;24(7):575-580. DOI: 10.5005/jp-journals-10071-23476.

3. Peng Q, Wang X, Zhang L, Chinese Critical Care Ultrasound Study Group (CCUSG). Using echocardiography to guide the treatment of novel coronavirus pneumonia. 2020;24(1):143. DOI: 10.1186/s13054020-02856-z.

4. World Health Organization. Transmission of SARS-CoV-2: implications for infection prevention precautions. In: Scientific brief. Geneva: World Health Organization; 2020, pp. 1-23.

5. Gargani L, Volpicelli G. How I do it: lung ultrasound. Cardiovasc Ultrasound 2014;12(1):25. DOI: 10.1186/1476-7120-12-25.

6. Tsutsui JM, Maciel RR, Costa JM, Andrade JL, Ramires JF. Hand-carried ultrasound performed at bedside in cardiology inpatient setting - a comparative study with comprehensive echocardiography. Cardiovasc Ultrasound 2004;2:24. DOI: 10.1186/1476-7120-2-24.

7. Mojoli F, Bouhemad B, Mongodi S, Lichtenstein D. Lung ultrasound for critically ill patients. Am J Respir Crit Care Med 2019;199(6):701-714. DOI: $10.1164 / \mathrm{rccm} .201802-0236 \mathrm{Cl}$.

8. Huang R, Poffenberger CM, Nguyen PD. Point-of-care ultrasound in austere environments a complete review of its utilization, pitfalls, and technique for common applications in austere settings. Emerg Med Clin 2017;35(2):409-441. DOI: 10.1016/J.EMC.2016.12.007.

9. Sorensen B, Hunskaar S. Point-of-care ultrasound in primary care: a systematic review of generalist performed point-of-care ultrasound in unselected populations. Ultrasound J 2019;11(1):31. DOI: 10.1186/ s13089-019-0145-4.

10. Lichtenstein DA. Lung ultrasound in the critically ill. Intensive Care Med 2004;30(2):183-184. DOI: 10.1007/s00134-003-2083-6.

11. Senniappan K, Sreedhar R, Babu MS, Dash PK, Gadhinglajkar SV, Sukesan S. Bedside lung ultrasound for postoperative lung conditions in cardiothoracic intensive care unit: Diagnostic value and comparison with bedside chest roentgenogram. Anesthesia 2019;13(4):649. DOI: 10.4103/aer.AER_125_19.

12. Guarracino F, Vetrugno L, Forfori F, Corradi F, Orso D, Bertini P, et al. Lung, heart, vascular, and diaphragm ultrasound examination of COVID-19 patients: a comprehensive approach. J Cardiothorac Vasc Anesth 2020;6:13. DOI: 10.1053/j.jvca.2020.06.013.

13. Kajimoto K, Madeen K, Nakayama T, Tsudo H, Kuroda T, Abe T. Rapid evaluation by lung-cardiac-inferior vena cava (LCl) integrated ultrasound for differentiating heart failure from pulmonary disease as the cause of acute dyspnea in the emergency setting. Cardiovasc Ultrasound 2012;10(1):49. DOI: 10.1186/1476-7120-10-49.

14. Gibson LE, Bittner EA, Chang MG. Handheld ultrasound devices: an emerging technology to reduce viral spread during the Covid-19 pandemic. Am J Infect Control 2020;48(8):968-969. DOI: 10.1016/j. ajic.2020.05.041.

15. ACR recommendations for the use of chest radiography and computed tomography (CT) for suspected COVID-19 infection. Available from: https://www.acr.org/Advocacy-and-Economics/ACRPosition-Statements/Recommendations-for-Chest-Radiographyand-CT-for-Suspected-COVID19-Infection

16. Khanji MY, Ricci F, Patel RS, Chahal AA, Bhattacharyya S, Galusko $V$, et al. The role of hand-held ultrasound for cardiopulmonary assessment during a pandemic. Prog Cardiovasc Dis 2020;63(5):690695. DOI: 10.1016/j.pcad.2020.07.003.

17. Qian F, Zhou X, Zhou J, Liu Z, Nie Q. A valuable and affordable handheld ultrasound in combating COVID-19. Crit Care 2020;24(1):334. DOI: 10.1186/s13054-020-03064-5. 